\title{
If All You Have Is A Hammer: TRIZ And Complexity
}

\author{
Darrell Mann ${ }^{1}$ \\ ${ }^{1}$ Systematic Innovation Network, Devon, UK \\ darrell.mann@systematic-innovation.com
}

\begin{abstract}
TRIZ is essentially a distillation of the 'first principles' of problem solving. It was originally developed for complicated technical problem and opportunity situations and, through ARIZ, has been deeply optimized for such roles. Increasingly, however, the world has become dominated by complex, non-technical situations, and in these environments many of the tools, methods and processes of traditional TRIZ become highly inappropriate. Complex situations are characterised by an absence of 'root-causes', have cause-effect relationships that are frequently tenuous, and are vulnerable to highly non-linear 'butterfly effects'. The paper describes the creation and verification of coherent non-linear, iterative divergent/convergent processes that take advantage of the first principle nature of TRIZ, and complement them with essential pieces from other domains of science. In a final section, the paper provides change agents with a menu of heuristics for determining the most appropriate TRIZ and non-TRIZ tools and strategies for any given situation.
\end{abstract}

Keywords: Complexity, Cynefin, Complexity Landscape Model, Cybernetics, Law Of Requisite Variety, ARIZ.

\section{Introduction}

Genrich Altshuller starts The Innovation Algorithm ${ }^{1}$ with the story of B.S. Egorov's journey to solve the problem of a winding machine to place coils of wire onto a small diameter toroidal transformer. In the story, Egorov is revealed to have already worked on what he thought was a similar problem, designing a machine to place coils onto an inductor for use in telephones. The problem was that the diameter of the toroidal transformer was significantly smaller than that of the telephone inductor coil, and unfortunately the system for 'threading' the wire with a needle, which was okay for the $(7 \mathrm{~mm}+)$ diameter of the inductor coils was no longer viable at the new smaller $(2 \mathrm{~mm})$ diameter. A 'simple' problem had become a 'complicated' one. Had the new toroidal transformer been designed with a $6 \mathrm{~mm}$ diameter, he would have been able to simply scale the existing solution to the new diameter. No calculations would have been required, and there was little need for any actual thinking. But because the new diameter was smaller beyond a scaling threshold level, the needle solution would not work any longer. A contradiction had appeared, and as such it had become necessary to partake of some creative thinking. The contradiction needed to be solved. Or - as actually happened Egorov had a flash of inspiration when he saw an analogous solution in another domain, 
and was subsequently able to translate that solution into something that worked for the new $2 \mathrm{~mm}$ diameter transformer. The 'complicated' problem was successfully solved.

One might imagine that, over the course of time, the need for even smaller diameter coils would emerge. Egorov's new solution would perhaps continue to work down to $0.5 \mathrm{~mm}$. Making a $1 \mathrm{~mm}$ coil would be 'simple'. But then another contradiction would emerge, and hence the problem would again become 'complicated'. This sequence of 'simple' scaling solutions followed by periodic arrival of 'complicated' contradiction problems would likely continue for some time. But, importantly, not forever. At some point, when the dimensions get small enough, the physics of the situation change. When - as we are beginning to see in the world of semi-conductor design - the physical dimensions have become so small that designers need to take into account quantum effects, the design challenge can be seen to have crossed another discontinuous problemtype boundary. A 'complicated' problem will, at small enough size scale, turn into a problem that is 'complex'. The point here being that when we cross the complicatedcomplex boundary, as when we cross the simple-complicated boundary, the method by which we need to tackle the problem makes a non-linear shift. These kinds of simpleto-complicated or complicated-to-complex phase boundary are important from the perspective of the tools and methods that problem solvers need to bring to bear if they are to make meaningful progress.

There are other ways that the toroidal transformer manufacture problem might cross the threshold from complicated into complexity. Traditional TRIZ was very much focused on technical problems. And moreover, the large majority of these technical problems turned out to be complicated. And so traditional TRIZ worked. In today's massively inter-connected world, however, it is increasingly rare that we find ourselves able to 'merely' focus on just the technical problem. The moment, for example, that we have to introduce our boss to the merits of our beautiful new contradiction-solving solution, we find ourselves having to acknowledge that bosses don't always do things for purely technical reasons. If the boss doesn't understand the new solution, there is the likelihood that they will become defensive. If the new solution causes the old solution to become redundant, and it was the boss who designed that old solution, their ego may easily become offended. If the boss is afraid of the next boss up the hierarchy, maybe they don't feel brave enough to argue the case for the new solution. Not to mention the customer, and what they might think about the risks associated with change. Do they trust us to deliver? The point, in any of these scenarios, is that our 'complicated' problem has become 'complex'. As soon as two or more human beings are present in the system, guaranteed it has become complex. People love change, but they hate being changed.

Increase the level of complexity sufficiently and we can easily find ourselves crossing another discontinuous, non-linear threshold. Complex systems, especially those involving humans and human behaviour have the occasional propensity to devolve into 'chaos'. Think of the devastation caused by Hurricane Katrina, or other extreme weather events, where quite clearly the 'recommended' rescue and recovery advice 
didn't work. When we find ourselves in chaos, it rapidly becomes clear that the usual 'rules' don't apply any more. The best thing we can do is either 'batten down the hatches' or get ourselves out of harm's way. Fortunately, chaos, because of its instability, tends not to last for very long. The hurricane passes. As far as Egorov's toroidal transformer situation is concerned, chaos could appear from a number of directions. The factory could burn down. Or the raw materials suffer a global shortage. Or we find ourselves subject to a public scandal and our main customer decides they don't wish to do business with us anymore.

Ultimately, the point of the discussion is this. A 'simple' problem is different to a 'complicated' one, is different to a 'complex' one, is different to a 'chaotic' one. Each demands a different strategy, methodology and potentially quite different tools if a viable set of solutions is to be delivered. Problem-solvers, in other words, if they are to stand the best chance of being successful, need to know what type of problem they are facing. It is to this identification issue that we now turn our attention.

\section{A Complexity Landscape}

The field of Complex Adaptive Systems (CAS) is still a relatively new one. Many authors and scientists are still caught in the struggle to find a coherent means of understanding and describing how problem-solvers and organisations might best operate under different complexity conditions. One of the most popular complex system frameworks is 'Cynefin'2. This framework divides the world into essentially the same four different types - simple (often labelled, 'obvious' in later iterations of the model), complicated, complex and chaotic - as used in the earlier description of the different variations of the Egorov toroidal transformer problem. As an orientation tool, Cynefin has found an audience mainly due to its elegant simplicity. If someone has never thought about what type of system they are operating within, it provides a ready supply of actionable insight. One of the main things it misses, however, is a means of describing the characteristics not just of a system under investigation, but also the characteristics and context of the surrounding environment within which that system is expected to operate. This shortfall has been addressed in the Complexity Landscape Model (CLM) reproduced in Figure 1.

More details of this Model can be found in an ongoing series of papers ${ }^{3,4}$ and so only the basics will be reviewed here. Essentially the CLM comprises a 2-dimensional, $4 \times 4$ segment graph featuring the four different states of a system being analysed (y-axis) and the four different possible states of its surrounding ('super-system') environment. Importantly, to reflect the Cynefin insight that a 'simple' system can easily find itself tipping directly into a chaotic state, the 'system' axis is sequenced in such a way that these two states are positioned adjacent to one another. A significant aspect of this adjacency is that the world of 'Operational Excellence' has taught managers that the most efficient organisations are the ones in which as much as possible of the work needing to be performed has been standardized. Over-standardization (often referred to as the 
'Dodo Effect'), however, means that what was eliminated from a system because it was thought to be 'waste' turns to have not been waste at all, but the resource needed to survive and thrive in turbulent times. When enterprises find themselves operating with a system that has become chaotic, they can be seen to have fallen past the Disintegration Line and into the Collapse Zone. Not surprisingly, this is an area of the Model organisations are well advised to avoid. The second noteworthy area of the Model is the Resilience Zone. The principle defining boundary for this Zone is the Ashby Line. The Ashby Line is named after the founding father of Cybernetics, W.Ross Ashby ${ }^{5}$ and specifically his Law Of Requisite Variety, which in simple terms states that if a system is to survive it must possess a level of achievable 'variety' greater than that of its surroundings: 'only variety can absorb variety'.

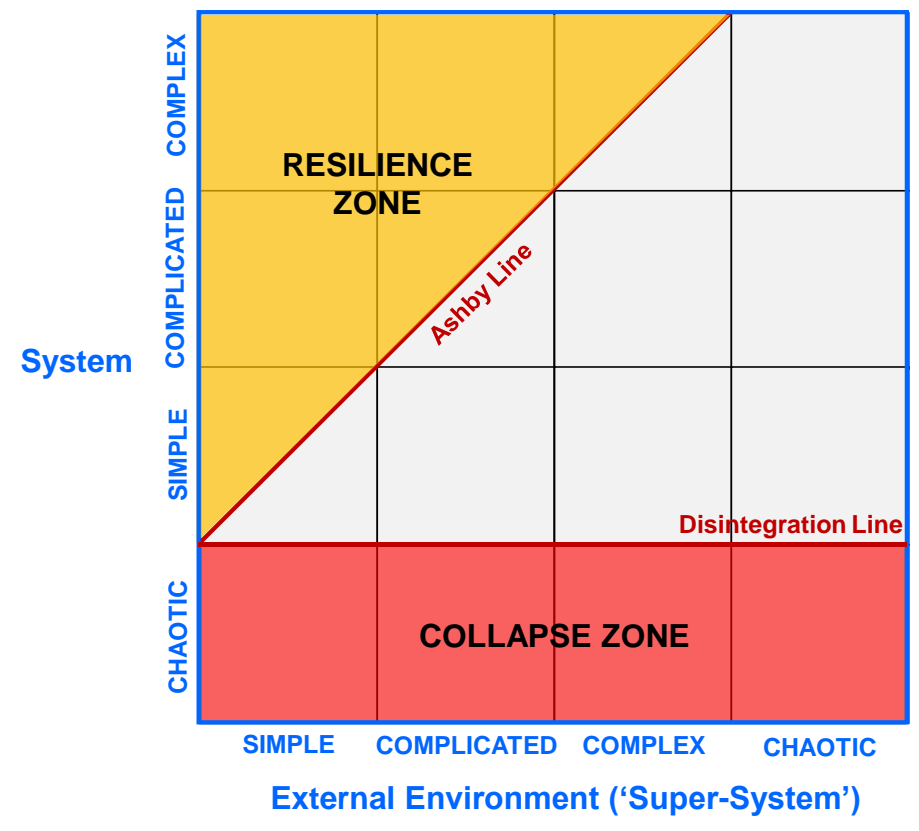

Fig. 1 Complexity Landscape Model (CLM)

The Resilience Zone, then, defines the region of the CLM where Ashby's Law is satisfied. This is the Zone where every enterprise should aspire to operate.

Figure 2 takes the basic CLM and plots a number of the previously described Egorov problem scenarios. It is worth describing these in a little more detail in the context of the Model:

- $\quad$ The knowledge to reliably manufacture telephone inductor coils already existed. Assuming the enterprise is stably manufacturing such designs, the situation can be mapped into the Simple-Simple segment, above the Ashby Line.

- As soon as the market (external environment) demanded a $2 \mathrm{~mm}$ diameter transformer, this couldn't be achieved by simply scaling the original solution, but 
rather required a contradiction to be solved. Prior to Egorov's solution, the problem can be seen to have shifted to the Complicated-Simple domain. In this domain, no solution was possible, and it was only when Egorov's mind started thinking about contradictions and solutions from other domains that a solution became possible. When the solution did finally arrive it was because Egorov had (unknowingly) crossed the Ashby Line and entered the Complicated-Complicated domain: the solution generation strategy was now commensurate with the problem.

- If we can imagine further reducing the size of the coil to the level where quantum effects have started to become relevant, then the problem will become a complex one. As with the 'complicated' version of the problem, we will again only be able to solve the problem if we move our problem-solving system up above the Ashby Line and into the Complex-Complex segment of the Model.

- A similar shift takes place if the problem becomes 'convincing the boss' of the merits of our technical solution. And, again, we will only make meaningful progress on this version of the problem if we move into the region of the ComplexComplex segment above the Ashby Line.

- Finally, if the factory burns down, while the fire is burning, everything shifts into the Chaos-Chaos region. There is no hope for 'resilience' at this stage, but rather we must act to put the fire out and, as quickly as possible see what can be done to get back into the Complex super-system column of the Model.

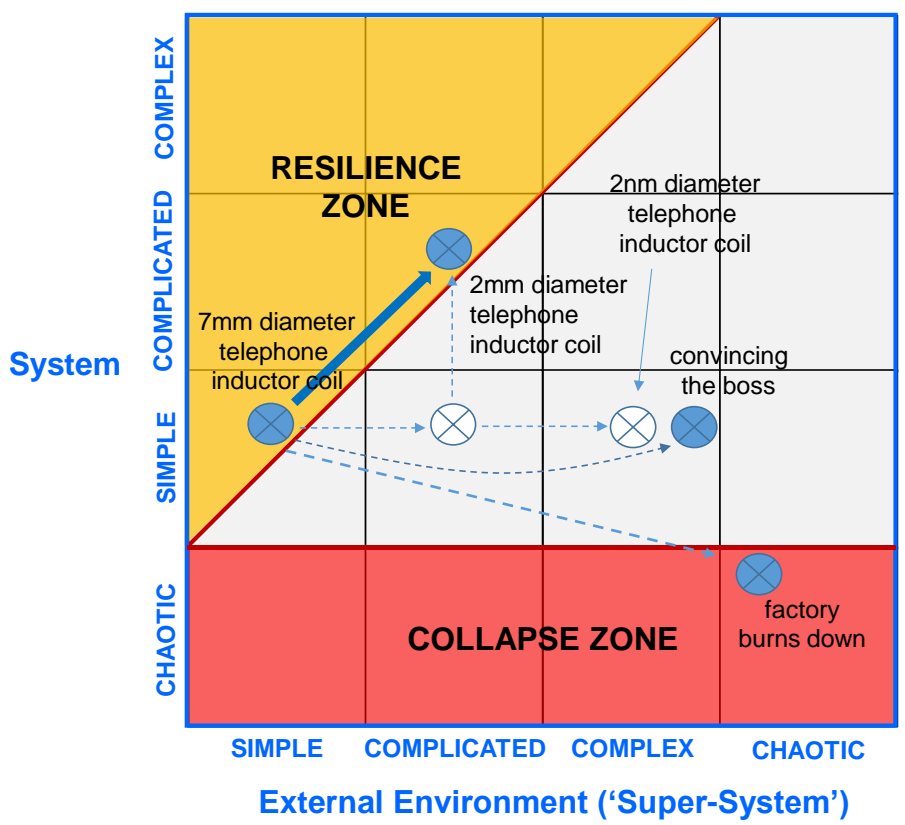

Fig. 2 Egorov Problem Scenarios Mapped Onto The Complexity Landscape Model 
Having now described how it is possible to map 'where' a problem sits on the Complexity Landscape, the next job is to establish the relevant solution approaches for each of the different scenarios. It turns out there are four that require step-change different shifts in approach:

1) Below the Ashby Line

2) Above the Ashby Line in the 'Simple' Super-System column

3) Above the Ashby Line in the 'Complicated' Super-System column

4) Above the Ashby Line in the 'Complex-Complex' triangle

The first two are the easiest to deal with. The third scenario is the one most amenable to 'traditional TRIZ' - as described in the Egorov toroidal transformer story. The fourth, although it appears to be a relatively small area of the overall Complexity Landscape Model is actually the one that is the most likely to be present. If only because the moment the 'system' under consideration includes humans - whether they be 'customers', the team working on the problem, the senior management of the enterprise or regulators - it will inherently have become a complex problem. This is the domain we will therefore be forced to spend the most time discussing.

\subsection{Below The Ashby Line}

The blinding-flash-of-the-obvious requirement when problem solvers find themselves in this situation is to alter the situation in a way that shifts it above the Ashby Line. Easy to say, not so easy to do. In no small part because the natural tendencies of the world serve to shift systems in the wrong direction. Figure 3 shows the two biggest 'natural' forces and the direction they act:

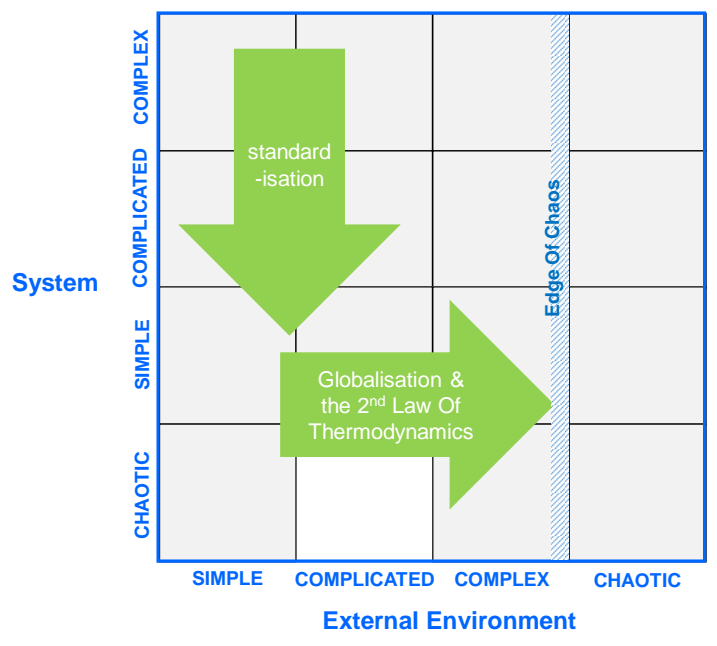

Fig. 3 'Natural' Forces Act Against Resilience 
(Whether 'standardisation' counts as a strictly 'natural' force is debatable. What is certain, however, is that ever since F. W. Taylor introduced 'Scientific Management' into the world of work in the early part of the $20^{\text {th }}$ Century, standardisation has been the primary motivation of almost every manager and leader on the planet.)

As far as the Second Law Of Thermodynamics is concerned ('disorder (entropy) increases'), it is perhaps some consolation that it doesn't lead inevitably to 'Chaos'. As discussed earlier, chaos is a very unstable state, and as such, once a chaotic episode settles down, systems return to the Complex domain. More often than not, sitting in a sub-domain known as the "edge of chaos"'

Figure 4 illustrates the human-instigated strategies that can be deployed to counter these natural forces in order to cross the Ashby Line:

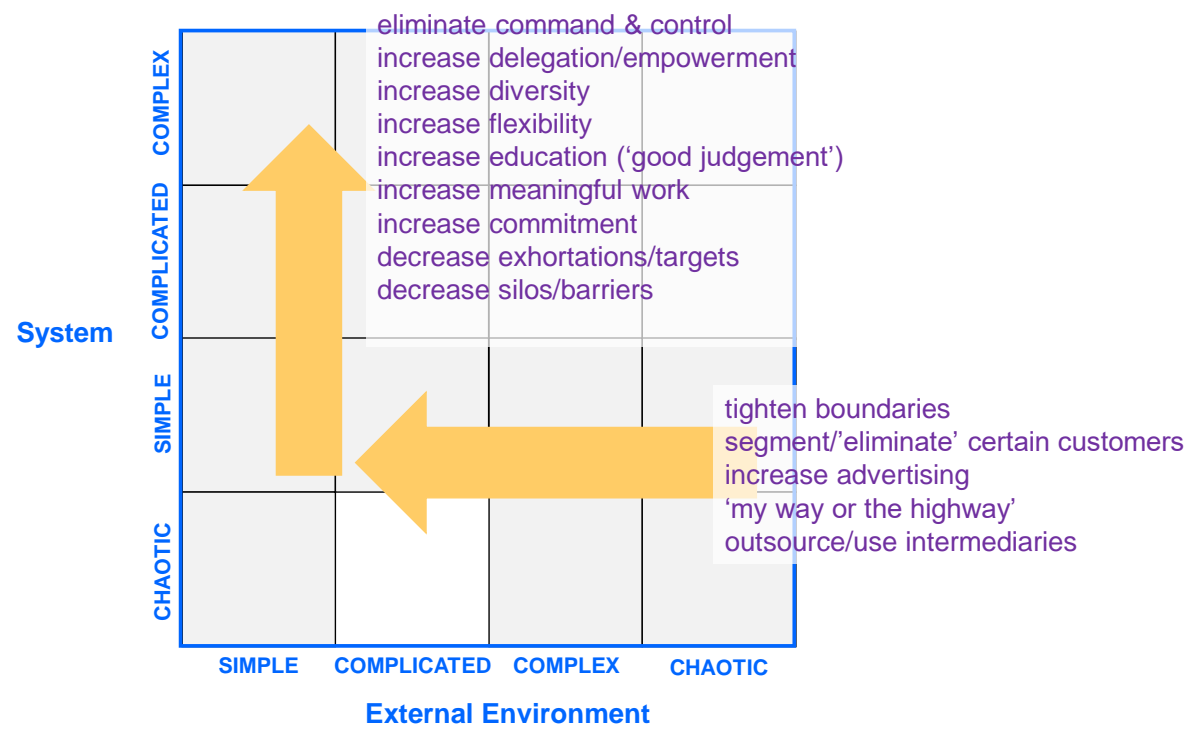

Fig. 4 Management Strategies For Shifting Into The Resilience Zone

\section{2 'Simple' And Above The Ashby Line}

This is the place to be when it comes to minimum problem-solving effort. When a problem solver is literally able to replicate an existing known solution, or simply scale from that existing solution, there is no need for any kind of creativity. Or for tools and methods like TRIZ. This is definitely the 'cookie-cutter' segment of the CLM. No innovation required here. So long as the system sits above the Ashby Line, it doesn't particularly matter how far above. Although, having said that, the further above the line it is - if the system is, for example, designed to operate as a 'complicated' or, better yet, a 'complex' entity - then the level of resilience is increased. The idea of an 'Ashby Margin'7 is something that organisations will shortly be able 
to objectively measure. The higher the margin, the more resilient the system will be, with, at the limit, the achievement of the ultimate level of resilience, 'antifragility's.

\section{3 'Complicated' And Above The Ashby Line}

Here is the domain of what might be thought of as 'traditional' TRIZ, whether at the level of individual tools, or overall start-to-finish processes like ARIZ. Complicated problems can be characterized in a number of different ways, but from a purely pragmatic perspective, there is a clear 'best' solution to the problem being addressed. Because this is the case, it is conceivable that the overall problem definition and solution generation process can be linear and sequential.

What this means in practical terms, too, is that it is appropriate for problem solvers to look for root causes, that there are clear connections between cause and effect, and that there are clear rules. Even if those rules are the TRIZ-based rules-for-breakingrules. Constructing a Function Analysis diagram and/or Su-Field Analysis model makes for the most effective and systematic ways of building the required understanding of the current system. From a classical TRIZ perspective, this problem type domain hits a clear sweet-spot, and even a moderately effective TRIZ user will very likely be able to devise a meaningful, practical solution.

\section{4 'Complex' And Above The Ashby Line}

The moment we acknowledge that a situation is complex, we have to accept that we are operating in conditions that are substantially different. There is no such thing as a 'root-cause' in a complex system, but rather a 'conspiracy' of causes. The links between cause and effect or often quite tenuous, and the behavior of the system becomes an emergent property. The Butterfly Effect further tells complex problem solvers that the world can easily become non-linear with apparently tiny perturbations quickly magnifying to become transformative. This means that things like the Pareto Effect no longer have a role. It is not possible to ignore anything that is currently 'insignificant' since it could easily be the weak-signal that triggers a complete disruption of the current system.

Because of these fundamental shifts in behavior, problem solvers need to adopt a different approach. The famous H.L. Mencken aphorism, 'For every complex problem there is a solution that is clear, simple and wrong' offers problem solvers a clear, but we now know outdated piece of advice when working with complexity. What we now know is that for every complex problem there are thousands of clear, simple wrong answers. But we know too that there is also the possibility of a 'right' one. Provided we are able to understand the behavior of a system from a first-principles level. The fact that the original TRIZ research (unknowingly) sought to distil the world of technology down to such first principles, means that the tools continue to have a good deal of relevance. The Inventive Principles are in effect the 'first principle' level array of solution generation possibilities; forcing problem solvers to examine systems through the lens 
of function forces first principles thinking; the value equation is a first principles distillation of the fundamental direction of travel of successful systems.

But - and this is a big but - just because TRIZ has given problem solvers an array of 'first principle' tools does not mean that when we connect these tools together into a process we can deal with a complex problem in a linear fashion. In this respect, the currently fashionable 'Design Thinking' world has much to teach problem solvers. At least from a methodological level. When the dust finally settles on the 2000+ Design Thinking texts presently in circulation, what complex problem solvers will likely be left with at the first principle level is the need for an overall process that is iterative and which contains alternating periods of divergent ('exploration') and convergent ('consolidation') effort. That overall process will likely as not look something like the one reproduced in Figure 5.

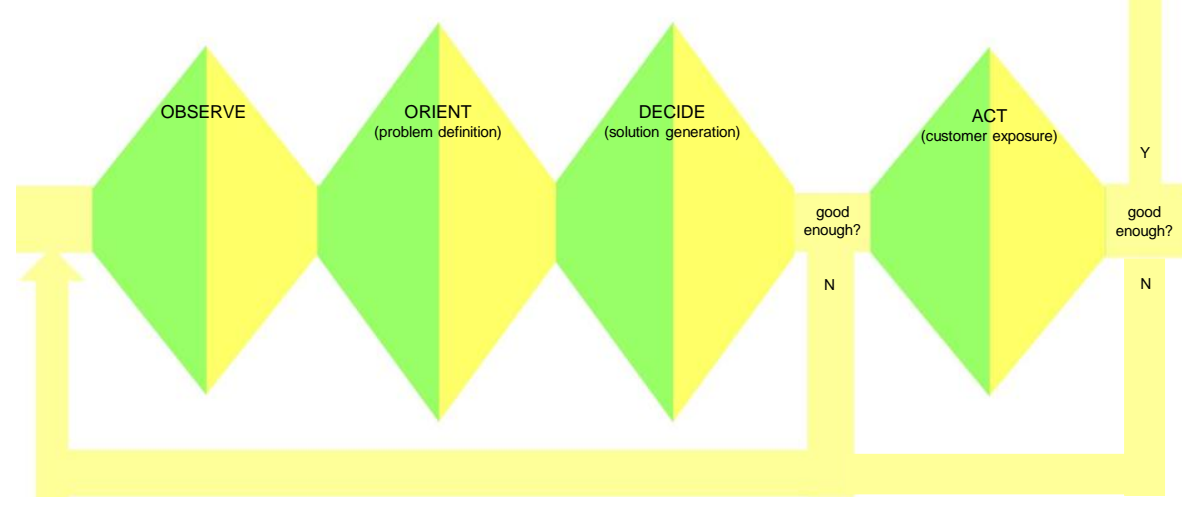

Fig. 5 Generic 'First Principle'-Level Complex Problem Solving Process

In true TRIZ, 'someone-somewhere-already-solved-your-problem' fashion, the benchmark for successful problem-solving in complex, edge-of-chaos situations can be found with the pilots of military fighter jets. The best-of-the-best in this domain was very likely Colonel John Boyd of the USAF. The model he developed is now known as the OODA-Loop. Observe-Orient-Decide-Act. The victor in any aerial dogfight, Boyd determined, was the one with the shortest OODA Loop cycle time? ${ }^{9}$.

Translated into complex business and technical problem-solving terms, OODA translates to Observing the problem context and surroundings, Problem Definition, Solution Generation, and Prototyping. The fourth stage being the intention is to get a physical prototype into the hands of the intended customer as quickly as possible in order to learn what aspects of that solution do and don't work for said customer. If (when) we learn a solution is not yet good enough, the process requires innovators to cycle back to the beginning of the process and conduct another iteration. 
Crucially, this cyclical process is built around the understanding that, first-principles or no, we can't actually 'know' what customers want until we deliver something to them and are able to obtain feedback from them when they're able to try something. In a complex environment, there is no such thing as the 'right' answer. Only 'right, rightnow'. Or, perhaps more precisely, 'fittest for purpose at this moment in time',

Also important to note is that the 'Systematic Innovation' methodology has been configured specifically with complex problems in mind. One of the basic tenets of SI is that, even though problem solvers can't know precisely what customers want, that doesn't mean that we can only make progress by trial and error. The TrenDNA toolkit ${ }^{10}$, for example, seeks to distill a first-principle understanding of human behavior into a repeatable method that, when iterated, allows problems solvers to not only shorten their OODA Loop cycle time, but also require fewer iterations.

Sitting right at the heart of this 'first principles' story from the situation understanding perspective is the s-curve, and the dynamics associated with the discontinuous shift from one s-curve to the next that occur when innovation takes place. The basic shape of the s-curve remains the same whether the system under consideration is complex or not. When the system is complex, however, the dynamics of the curve are dictated by the presence of virtuous and vicious cycles. Virtuous cycles begin with weak signals that then take advantage of positive feedback effects. Figure 6 illustrates one such virtuous cycle. One that, over the course of the last ten years has seen the rise to dominance of the Magnificent Seven Big Data organisations ${ }^{11}$.

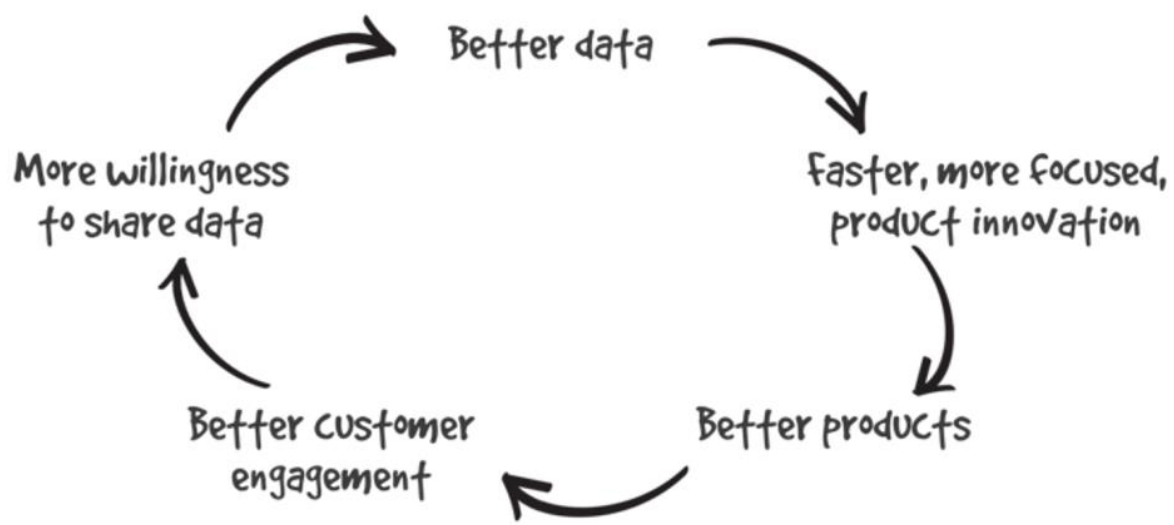

Fig. 6 Virtuous Cycle Responsible For The Rise Of The Magnificent Seven

At the other end of the s-curve come the vicious cycles. An illustration of the impact of vicious cycles on the failure of complex systems can be found in a recent analysis of the UK Brexit debacle ${ }^{12}$. The primary point of that paper was to register the need to break a vicious cycle as the only way to break out of the ever-more rapid tailspin into chaos. Breaking vicious cycles again becomes a task well suited to the TRIZ Contradiction tools. TRIZ is unable to reveal the vicious cycles (rather the Perception Mapping 
process ${ }^{13}$ has become the go-to method for doing this job), but once they are revealed, only TRIZ provides the wherewithal to break them and point the way towards the possible step-change jumps to the next s-curve.

\section{Conclusions: The Overall Role Of TRIZ}

98\% of all innovation attempts end in failure. 98\% of all TRIZ-originated innovation attempts end in failure. $98 \%$ of all Design-Thinking-originated innovation attempts end in failure. The same $98 \%$ figure applies to almost every problem-solving tool, method or strategy available to prospective innovators.

$98 \%$ of innovation attempts fail because $100 \%$ of innovation problems are complex and $98 \%$ of the people tasked with conducting the work either didn't understand that or weren't using tools commensurate with the complexity.

So what did the $2 \%$ of successful attempts do? The clear answer is that they knowingly - or, occasionally, accidentally - found themselves in the right place at the right time with the right solution at the right price for the right customer. Getting all of these things right at the same time is a challenging task for which there is no 'formula', other than a recognition that the innovation project needs to be coordinated according to the demands of the Figure 7 'Golden Triangle':

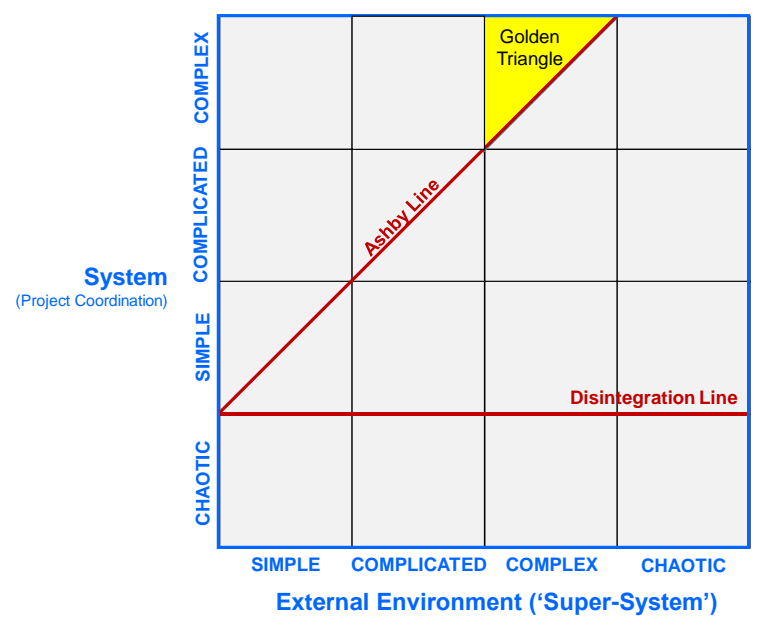

Fig. 7 'The Golden Triangle’ Of Successful Innovation Project Coordination

Which in practice means the following:

Tangible skills:

Systems Thinking

Thriving in complexity - rapid-learning cycles, 'first principles', s-curves, patterns, 'critical mass at the critical point'

Understanding the customer/consumer say/do gap 
Measuring what's important (rather than merely easy)

Having a clear compass heading

Knowing how to abstract problems to tap into solutions in other domains

('someone, somewhere already solved your problem')

Solving trade-offs \& compromises

Intangible skills:

Innovation is largely about the individual \& cultural intangibles

('Everyone has a plan until they get hit in the face' Mike Tyson)

Influencing others/ working together in cross-disciplinary teams

Persistence/bloody-mindedness/willingness to stick-with-difficult-stuff

Learning to live with continual 'failure'

Acknowledging that 'ideas' have zero value

The Progress Principle ${ }^{14}$

Because TRIZ has effectively created a 'database' of first-principle level understanding of what success in technical problem solving looks like, it has a necessary but not sufficient role in enabling prospective innovators to have the best chance of ending up in the $2 \%$ of successful projects. Much of the original TRIZ research was performed on technical problems falling in to the 'complicated' category, and this is the domain where the tools are at their most effective. TRIZ continues to be relevant in the 'complex' domain too, provided that users deploy the various different tools in an appropriate fashion rather than in the 'traditional' manner. What this means in practice demands more space than is available in this paper. That said, there are a number of (first-principle) heuristics that innovators and problem-solvers working in the Complex domain can readily adopt. In no particular order:

a) The 'traditional' TRIZ approach encourages users to progressively converge on 'the' contradiction and 'the' right Inventive Principle or Separation strategy, but this convergent-only trajectory makes no sense in the complex environment. Divergent parts of the process demand that users generate as many solution 'clues' as they can from as many Inventive Principles or Inventive Standards, or whatever other solution generation triggers the users have the time to work through. 'Diverge until it hurts' is a good heuristic. The eventual solution to a complex problem will almost inevitably emerge from a combination of partial solutions. The more partial solutions there are, the more likely it is that the necessary components of the winning combination will be present.

b) The Trends and particularly the Evolution Potential version thereof are often referred to in the Classical TRIZ world as 'advanced'. When used in the Complex domain, however, the Trends are more frequently observed to be 'easy' since they are well-suited to the divergent idea-generation task. The TRIZ Trends effectively tell problem solvers what the answers are, without having to know what the problem being solved necessarily is. This 180degree switch is quite counter-intuitive in the 'Complicated' world, but rapidly becomes the most sensible way of moving forward in the 'Complex' world. Each Trend 
jump effectively points the way to the next s-curve on the road to the Ideal Final Result solution...

c) ...while apparently being one of the simplest tools in the TRIZ toolkit, the Ideal Final Result can rapidly come to cause problems in the 'Complicated' world. This is because it is almost inevitable that the IFR looks nothing like the solution that an organisation is currently selling to customers. And moreover, if it is ideal it will also be 'free', which then necessitates not just technical innovation but a host of business innovations to accompany it. And herein lies an enormous challenge for the large majority of all enterprises operating in the $21^{\text {st }}$ Century: while the skills to innovate technically are well on their way to becoming a 'science', in the corresponding world of business and management, the innovation skills are still almost non-existent. The IFR tool is a tool for projects being coordinated in the Golden Triangle.

d) Whether the word 'TRIZ' survives into the long-term future continues to be a matter of some considerable uncertainty. What will inevitably prevail, however, is the contribution made regarding the importance of revealing and resolving contradictions. Contradiction resolution is the primary mechanism for innovation. The Contradiction tools and methods were developed for complicated problems, but they apply to complex ones equally well. They are also the most effective means of shifting a project from a position below the Ashby Line to one within the Resilience Zone. Resilience, in almost all ways, comes from the ability of an enterprise to solve contradictions faster than their competitors.

\section{References}

1. Altshuller, G., 'The Innovation Algorithm: TRIZ, Systematic Innovation And Technical Creativity', Technical Innovation Center, Worcester, MA, $1^{\text {st }}$ Edition, 1999.

2. Snowden, D.J., Boone, M.E., 'A Leader's Framework For Decision Making', Harvard Business Review, November 2007.

3. Mann, D.L., 'A Complexity Landscape', www.darrellmann.com, 31 March 2019.

4. Mann, D.L., 'The Resilience Zone', Systematic Innovation E-Zine, Issue 205, April 2019.

5. Ashby, W.R., 'An Introduction to Cybernetics', Chapman \& Hall, London, 1957, available electronically at http://pespmc1.vub.ac.be/books/IntroCyb.pdf.

6. Pascale, R.T., Millemann, M., Gioja, L., Surfing The Edge OF Chaos: The Laws Of Nature And The New Laws Of Business', Crown Business, 2000.

7. Mann, D.L., 'Calculating Ashby Margin', SI E-Zine, Issue 206, May 2019.

8. Taleb, N.N., 'AntiFragile: Things That Gain From Disorder', Random House, 2012.

9. Richards, C., 'Certain To Win: The Strategy Of John Boyd Applied To Business', Xlibris Corp, 2004.

10. Mann, D.L., Özözer, Y., 'TrenDNA: Understanding Populations Better Than They Understand Themselves', IFR Press, 2009.

11. Mann, D.L., 'The Magnificent Seven Versus The Insurance Industry', keynote address, Cardiff University Business School, 19 February 2019.

12. Mann, D.L., 'Unravelling The Brexit Debacle', EMBA Lecture Notes, September 2017. 
13. Mann, D.L. 'Hands-On Systematic Innovation For Business \& Management', IFR Press, 2009.

14. Amabile, T., Kramer, S., 'The Progress Principle: Using Small Wins To Ignite Joy, Engagement, And Creativity At Work', Harvard Business Review Press, 2011. 